\title{
Electrical Signal-to-Noise Ratio Enhancement in Detection of Mid-IR Signals by Four-Wave Mixing in As2S3-on-LiNb03 Waveguides
}

\author{
Qi Chen, Christi Madsen \\ Department of Electrical \& Computer Engineering, Texas A \& M University, College Station, TX, USA \\ Email: chenqicage21@tamu.edu
}

Received 9 April 2016; accepted 16 May 2016; published 19 May 2016

Copyright (C) 2016 by authors and Scientific Research Publishing Inc.

This work is licensed under the Creative Commons Attribution International License (CC BY). http://creativecommons.org/licenses/by/4.0/

(c) (i) Open Access

\begin{abstract}
Mid-infrared (mid-IR) signals can be converted to near-infrared (near-IR) wavelengths in $\mathrm{As}_{2} \mathrm{~S}_{3^{-}}$ on- $\mathrm{LiNbO}_{3}$ waveguides by high efficiency four-wave mixing. It provided us a solution to detect mid-IR signals indirectly by state-of-the-art near-IR detectors. High efficiency four-wave mixing was demonstrated and electrical signal-to-noise ratio (eSNR) improvement was also investigated. Compared to direct detection by PbSe and HgCdTe (MCT) mid-IR detectors, the calculation indicated that, at room temperature, the indirect detection to mid-IR signals increased the electrical signal-to-noise ratio up to $67 \mathrm{~dB}$.
\end{abstract}

\section{Keywords}

Chalcogenide, Mid-Infrared, Four-Wave Mixing

\section{Introduction}

There is a wide variety of mid-IR applications in different fields, including environment, medicine and military. Mid-IR detectors are extremely limited in terms of operation condition, noise performance and speed [1]. On the contrary, state-of-the-art near-IR detectors have advantages in these aspects. Since detectors at near-IR wavelengths exhibit superior performances in speed, noise and sensitivity, the indirect detection scheme is promising to improve the detection performance [2]. $\mathrm{As}_{2} \mathrm{~S}_{3}-\mathrm{on}-\mathrm{LiNbO}_{3}$ waveguides can provide an excellent solution to wavelength conversion by four-wave mixing.

Four-wave mixing (FWM) is a third-order nonlinear process. In degenerate FWM, if the phase-matching con- 
dition is met, two pump photons and a signal photon will generate an idler photon. Previously, we successfully fabricated low-loss $\mathrm{As}_{2} \mathrm{~S}_{3}$-on- $\mathrm{LiNbO}_{3}$ for mid-IR at $4.8 \mu \mathrm{m}$ [3]-[7]. Taking advantage from it, our phasematched $\mathrm{As}_{2} \mathrm{~S}_{3}$-on- $\mathrm{LiNbO}_{3}$ waveguides can be utilized to achieve high efficiency FWM [8], which converts 4.6 $\mu \mathrm{m}$ mid-IR signals to $1.32 \mu \mathrm{m}$ for indirect detection. With high parametric conversion efficiency, significant electrical signal-to-noise ratio improvement can be expected.

In this paper, we demonstrated high efficiency FWM design in $\mathrm{As}_{2} \mathrm{~S}_{3}$-on- $\mathrm{LiNbO}_{3}$ waveguides with thin $\mathrm{MgF}_{2}$ cladding on top. Under $0.1 \mathrm{GW} / \mathrm{cm}^{2}$ pump power intensity at $2.05 \mu \mathrm{m}$, mid-IR signals at $4.6 \mu \mathrm{m}$ can be converted to $1.32 \mu \mathrm{m}$ near-IR signals with $-10.5 \mathrm{~dB}$ parametric conversion efficiency. In order to analyze the eSNR improvement in indirect detection, we calculated the noise performance in detection of $4.6 \mu \mathrm{m}$ mid-IR signals by using PbSe and MCT mid-IR detectors and In GaAs near-IR detector after an $\mathrm{As}_{2} \mathrm{~S}_{3}$-on- $\mathrm{LiNbO}_{3}$ wavelength converter. Our calculation showed that the indirect detection to mid-IR signals can increase the eSNR up to 67 $\mathrm{dB}$, which is $17 \mathrm{~dB}$ higher than the results in silicon waveguides in [2].

\section{Four-Wave Mixing}

Figure 1 illustrates the indirect detection of mid-IR signals converted to near-IR wavelengths by FWM and detected by near-IR detectors.

In order to achieve high efficiency FWM, phase-matching condition has to be satisfied. Figure 2 shows the phase mismatch curve for our $\mathrm{As}_{2} \mathrm{~S}_{3}$-on- $\mathrm{LiNbO}_{3}$ waveguide design, where $\mathrm{As}_{2} \mathrm{~S}_{3}$ is $1.42 \mu \mathrm{m}$ wide and $2.7 \mu \mathrm{m}$ height with $0.089 \mu \mathrm{m} \mathrm{MgF}$ cladding on top. $\mathrm{MgF}_{2}$, which has wide transmission range $(0.2 \mu \mathrm{m}-7 \mu \mathrm{m})$ at mid-IR, is a good candidate for cladding material because of its ruggedness and durability. It also keeps the potential of optical tuning by adding electrodes on it [8]. Since the phase-matching condition is met at $1.32 \mu \mathrm{m}, 4.6$ $\mu \mathrm{m}$ and the region around the pump wavelength, which means that this design can provide high efficiency FWM process.

The parametric conversion efficiency $G_{i}$ is described by Equation (1) as below:

$$
G_{i}=\left[\frac{\gamma P_{p}}{g} \sinh \left(g L_{\text {eff }}\right)\right]^{2}
$$

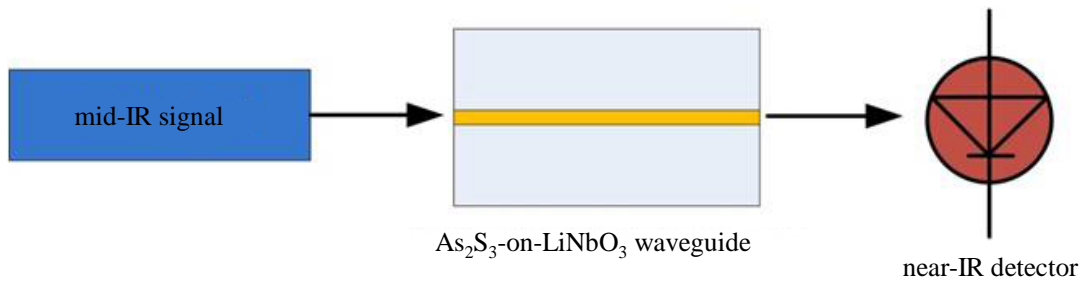

Figure 1. Schematic diagram of indirect mid-IR detection.

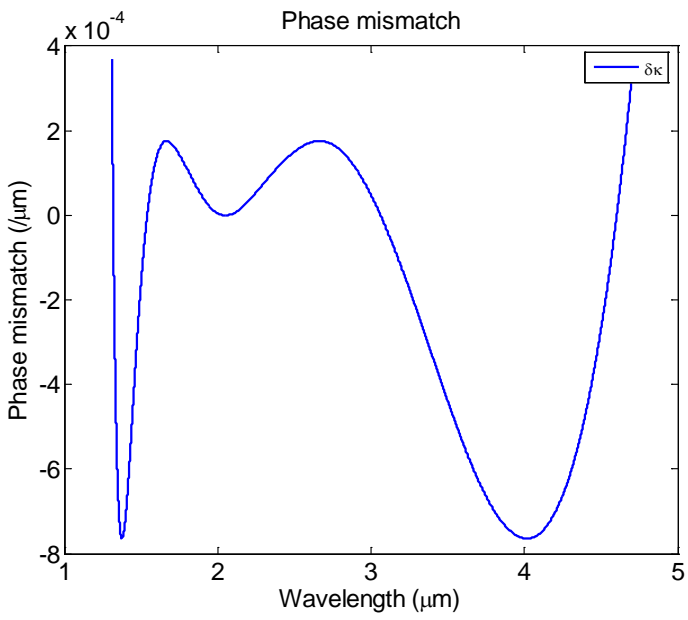

Figure 2. Phase mismatch. 
where $g$ is parametric gain, $\gamma$ is propagation constant and $\mathrm{L}_{\text {eff }}$ is the $\mathrm{As}_{2} \mathrm{~S}_{3}$-on- $\mathrm{LiNbO}_{3}$ waveguide length for nonlinear process. They can be expressed by Equation (2)

$$
g=\sqrt{\left(\gamma P_{p}\right)^{2}-(\Delta \kappa / 2)^{2}}
$$

where $\Delta \kappa$ is phase mismatch. The parametric conversion efficiency $G_{i}$ is shown in Figure 3 . When $L_{e f f}$ is $4 \mathrm{~cm}$ and $P_{p}$ is $0.1 \mathrm{GW} / \mathrm{cm}^{2}$, the parametric conversion efficiency is $-10.5 \mathrm{~dB}$.

\section{Noise}

Basically, there are two major types of noises in indirect detection associated with wavelength conversion, including pump transferred noise and quantum noise. Since the noise transfer from pump is considered to be detrimental for large signal powers [9] [10], it is negligible for weak mid-IR signals in our calculations. However, quantum noise should be considered in a parametric process like FWM and it will be transferred to In GaAS photodetector as quantum fluctuation, $i_{q t}^{2}$, described by Equation (3) as below.

$$
\frac{i_{q t}^{2}}{B_{e}}=4 R^{2} G_{i} P_{i n} \frac{h c}{2 \lambda}\left(2 G_{i}+1\right)
$$

where $h$ is Planck constant and c is speed of light. $R$ is responsivity and $B_{e}$ is the electrical bandwidth of In GaAs photodetector in the specification sheet. Pin is incident mid-IR signal power and $G_{i}$ is parametric conversion efficiency in Equation (1). Since weak mid-IR signal is considered, we use Pin $=1 \mu \mathrm{W}$ with $1 \mathrm{GW} / \mathrm{cm}^{2}$ pump power.

Furthermore, there are some intrinsic noises from the detector itself, including thermal noise and shot noise. Thermal noise results from random thermal motion of electrons in a resistor manifests as a fluctuating current, which is described by Equation (4).

$$
\frac{i_{t h}^{2}}{B_{e}}=4\left(k T / R_{L}\right) F_{n}
$$

where $k$ is Boltzmann constant, $T$ is operating temperature, $R_{L}$ is load resistance and $F_{n}$ is circuitry noise figure. Shot noise is a manifestation of the fact that an electric current consists of a stream of electrons that are generated at random times, shown is Equation (5)

$$
\frac{i_{s h}^{2}}{B_{e}}=2 q\left(R G_{i} P_{i n}+i_{d}\right)
$$

where $q$ is elementary charge and $i_{d}$ is dark current. Since the noise components are characterized, we can simply calculate the noises for indirect detection of mid-IR signals. The parameters used in our calculation are shown in Table 1, which comes from state-of-the-art commercial near-IR and mid-IR detectors in [10] and [11].

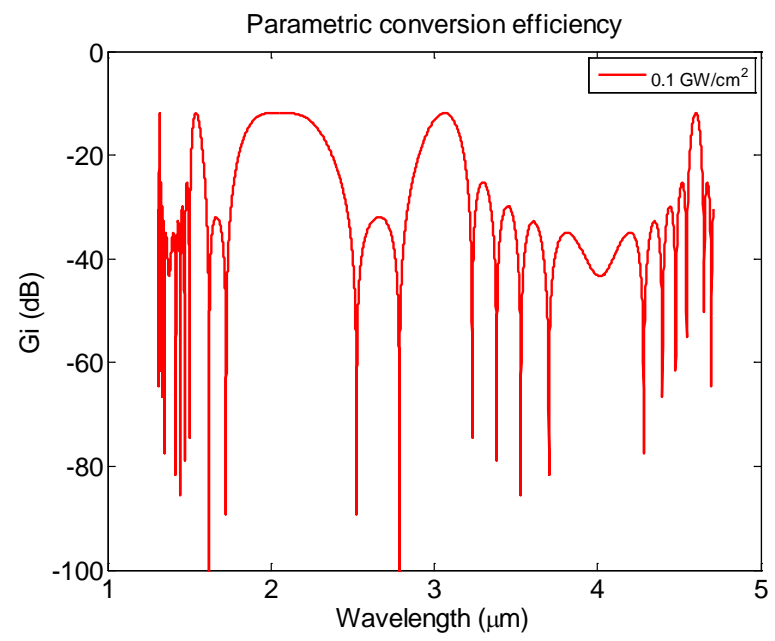

Figure 3. Parametric conversion efficiency. 
Table 1. Detector parameters.

\begin{tabular}{cccccc}
\hline & $\lambda(\mu m)$ & $T(K)$ & $R$ & $R_{L}(\Omega)$ & $i_{d}(p A)$ \\
\hline InGaAs & 1.55 & 300 & $0.95 \mathrm{~A} / \mathrm{W}$ & $8 \mathrm{M}$ & 80 \\
PbSe & 4.6 & 300 & $3 \times 10^{3} \mathrm{~V} / \mathrm{W}$ & $0.3 \mathrm{M}$ & NA \\
MCT & 4.6 & 300 & $3 \times 10^{3} \mathrm{~V} / \mathrm{W}$ & $1.5 \mathrm{k}$ & NA \\
\hline
\end{tabular}

Figure 4 demonstrates that the noise from InGaAs photodetector at near-IR by FWM at room temperature with incident power of $1 \mu \mathrm{W}$. It is obvious that the eSNR of the detector is limited by shot noise at low pump power intensities. However, when pump power intensity increases over $0.25 \mathrm{GW} / \mathrm{cm}^{2}$, the parametric conversion efficiency increases and therefore, the quantum noise becomes dominant. At room temperature, thermal noise is little and it can be further improved by cooling of near-IR detector in indirect detection process using thermoelectric cooling (TEC).

Since the noise performance of the near-IR detector is well demonstrated, in order to make comparison for eSNR, it is essential to characterize the mid-IR detectors. There are three types of commercial mid-IR detectors: thermal detectors, photovoltaic (PV) and photoconductive (PC) detectors. Thermal detectors are based on the resistance change by incident mid-IR signals. They have low sensitivity and are also too slow for real-time detection. The operational principle of PV detectors is generating current by absorption of photons with energy beyond their bandgap. Due to the narrow bandgap of mid-IR photons, the excess thermal generation during the detection will lead to considerable dark current and then large shot noise. It is necessary to operate them at low temperature $(\sim 70 \mathrm{~K})$ to reduce dark current. In other words, PV detectors are limited by its shot noise level at room temperature. Therefore, only PC detectors are characterized here.

In terms of PC detectors, the voltage change is measured, which results from the conductivity change of the medium by photo-induced carriers. The major noise components of PC detectors include thermal noise, background noise, generation-recombination (G-R) noise and flicker noise. Since a low-pass filter can be used in the system to eliminate flicker noise, the total noise will consist of thermal noise, background noise and G-R noise. As above, Thermal noise is described in Equation (4). Background noise is shown in Equation (6)

$$
\frac{i_{b g}^{2}}{B_{e}}=q^{2} \eta A E_{B}
$$

where $\eta$ is quantum efficiency and $A$ is active detector area. Photon flux density EB is described in Equation (7)

$$
E_{B}=\int_{0}^{\lambda_{c}} \varepsilon_{\lambda} \frac{2 \pi c}{\lambda^{4}}\left[\frac{1}{e^{h c / \lambda k T}-1}\right] d \lambda
$$

where $\varepsilon_{\lambda}$ is emissivity for the medium material and $\lambda_{c}$ is cut-off wavelength.

Currently, there are two kinds of commercial PC detectors: PbSe and MCT detectors. In view of relative slow response of mid-IR detectors, a $0.1 \mathrm{MHz}$ electrical bandwidth is used. Based on the calculations, thermal noise is dominant in PbSe and MCT detectors. For the same $1 \mu \mathrm{W}$ incident power at mid-IR, the total noise of PbSe and MCT detectors are $0.01 \mathrm{pA}^{2} / \mathrm{Hz}$ and $16.73 \mathrm{pA}^{2} / \mathrm{Hz}$, respectively.

\section{4. eSNR Improvement}

The eSNR improvement is defined by comparing the eSNR of InGaAs photodetector to those of PbSe and MCT mid-IR photodetectors, as described by Equation (8)

$$
e S N R_{\text {near-IR }} / e S N R_{\text {mid }-I R}
$$

In order to analyze eSNR enhancement by indirect detection to weak mid-IR signals, we used different input power levels at $0.1 \mu \mathrm{W}, 1 \mu \mathrm{W}$ and $10 \mu \mathrm{W}$ in calculations to estimate the detection limit, as presented in Figure 5 . Under the same incident power level, the eSNR improvement with respect to PbSe detectors is larger than that with respect to MCT detectors. When the input mid-IR power is $0.1 \mu \mathrm{W}$, the largest eSNR improvement is achieved with respect to both PbSe and MCT detectors, which are $67 \mathrm{~dB}$ and $32 \mathrm{~dB}$, respectively. In addition, compared to different incident power levels, indirect detection is more advantageous at lower input levels. 


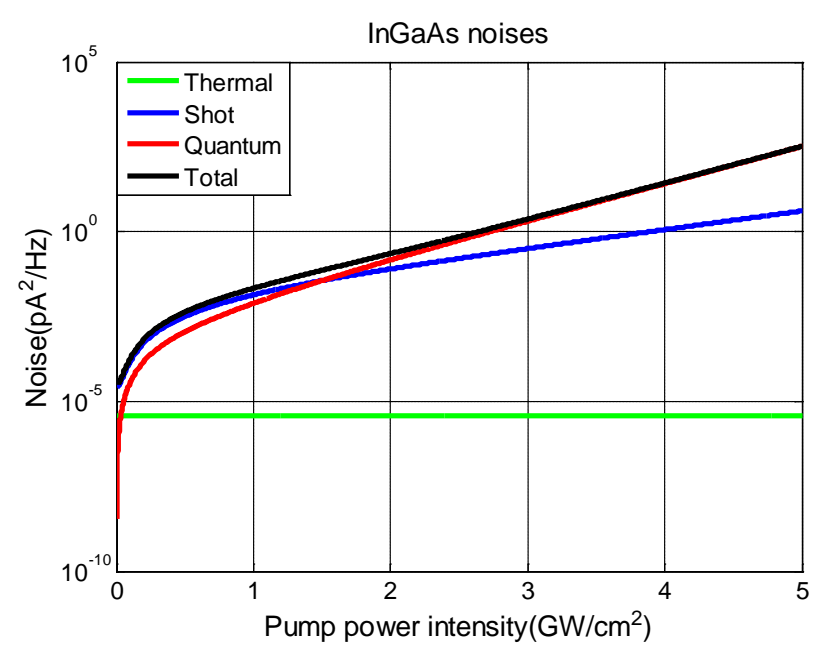

Figure 4. InGaAs noise at near-IR for indirect detection.

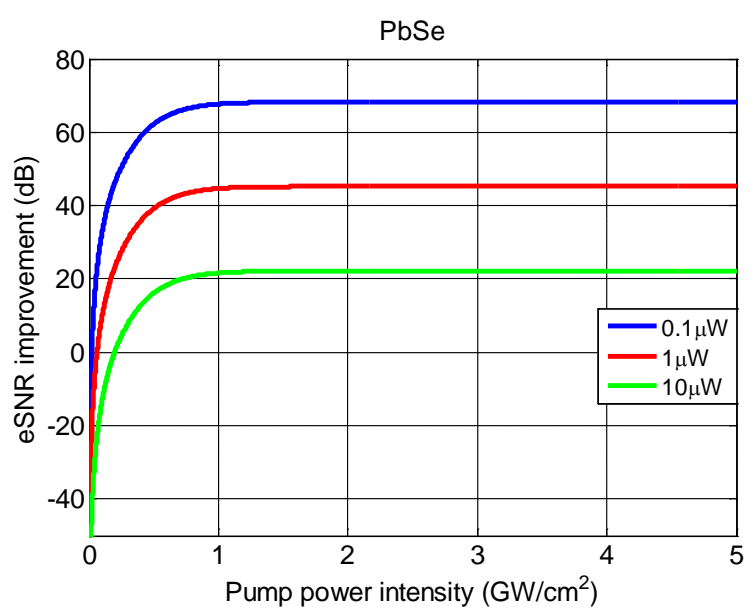

(a)

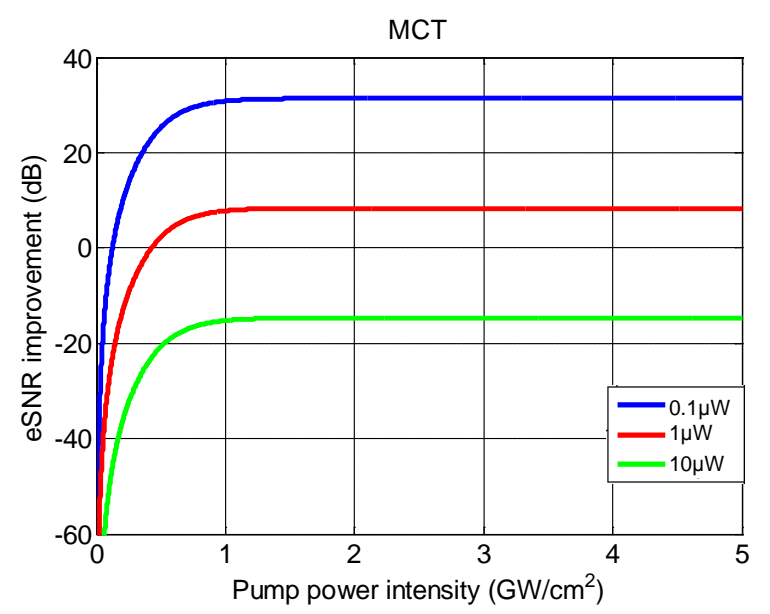

(b)

Figure 5. (a) eSNR enhancement compared to PbSe photodetectors; (b) eSNR enhancement compared to MCT photodetectors.

It is obvious that the eSNR enhancement increases with the increasing pump power intensity until it reaches 1 $\mathrm{GW} / \mathrm{cm}^{2}$, at which eSNR enhancement saturates resulting from the limit of quantum noise to FWM process. The phenomenon indicates that although the parametric conversion efficiency increases with the increasing pump power intensity, it is cancelled out by the increasing quantum noise. Furthermore, if we don't use TEC system, power radiation will increase thermal noise by heating the medium material. In this case, the pump power is supposed to be controlled in a proper range. Therefore, the maximized eSNR enhancement can be achieved.

\section{Conclusion}

In summary, we demonstrate phase-matched $\mathrm{As}_{2} \mathrm{~S}_{3}$-on- $\mathrm{LiNbO}_{3}$ waveguide design, which is able to convert 4.6 $\mu \mathrm{m}$ mid-IR signals to $1.32 \mu \mathrm{m}$ near-IR light for indirect detection. We analyze and compare noise performance for PbSe and MCT mid-IR detectors and InGaAs near-IR detector. Our calculations show that the eSNR improvement by indirect detection of mid-IR signals is $17 \mathrm{~dB}$ larger than the results in silicon waveguides in [2]. This improvement is more advantageous in detection to weak signals.

\section{Acknowledgements}

This material is based upon work supported by the National Science Foundation under grant No. EEC-0540832. 


\section{References}

[1] Characteristics and Use of Infrared Detectors. Solid State Division, Hamamatsu Photonics K.K, Tokyo, 2004.

[2] Huang, Y., Tien, E.K., Gao, S., Kalyoncu, S.K., Song, Q., Qian, F., Adas, E., Yildirim, D. and Boyraz, O. (2011) Electrical Signal-to-Noise Ratio Improvement in Indirect Detection of Mid-IR Signals by Wavelength Conversion in Silicon-on-Sapphire Waveguides. Applied Physics Letters, 99, 181122. http://dx.doi.org/10.1063/1.3651292

[3] Xia, X., Chen, Q., Tsay, C., Arnold, C.B. and Madsen, C.K. (2010) Low-Loss Chalcogenide Waveguides on Lithium Niobate for the Mid-Infrared. Optics Letters, 35, 3228-3230.

[4] Tan, W.C., Chen, Q., Kim, J. and Madsen, C. (2011) A Hybrid $\mathrm{As}_{2} \mathrm{~S}_{3}$ Mach-Zehnder Interferometer Prepared by Magnetron Sputtering and its Photodarkening Effect. IEEE Journal of Quantum Electronics, 48, 237-243.

[5] Madsen, C.K., Chen, Q., Kim, J.H. and Zhou, Y. (2013) Photonic Processing Using Integrated Optical Filters. Optical Fiber Communication Conference. http://dx.doi.org/10.1364/ofc.2013.oth4d.3

[6] Zhou, Y., Xin, X., Snider, W.T., Kim, J.H., Chen, Q., Tan, W.C. and Madsen, C.K. (2011) Two-Stage Taper Enhanced Ultra-High Q $\mathrm{As}_{2} \mathrm{~S}_{3}$ Ring Resonator on LiNbO3. IEEE Photonics Technology Letters, 23, 1195-1197. http://dx.doi.org/10.1109/LPT.2011.2158414

[7] Zhou, Y., Snider, W.T., Chen, Q., Kim, J.H. and Madsen, C.K. (2011) Ultrahigh Q Long-Path $\mathrm{As}_{2} \mathrm{~S}_{3}$ Ring Resonator on $\mathrm{LiNbO}_{3}$. CLEO: Applications and Technology, Optical Society of America, JTuI9.

[8] Chen, Q., Wang, X. and Madsen, C. (2012) Phase-Matching and Parametric Conversion for the Mid-Infrared in As2S3 Waveguides. Optics and Photonics Journal, 2, 260-264. http://dx.doi.org/10.4236/opj.2012.24031

[9] Kylemark, P., Hedekvist, P.O., Sunnerud, H., Karlsson, M. and Andrekson, P.A. (2004) Noise Characteristics of Fiber Optical Parametric Amplifiers. Journal of Lightwave Technology, 22, 409. http://dx.doi.org/10.1109/JLT.2003.822152

[10] Moro, S., Peric, A., Alic, N., Stossel, B. and Radic, S. (2010) Phase Noise in Fiber-Optic Parametric Amplifiers and Converters and Its Impact on Sensing and Communication Systems. Optics Express, 18, 21449. http://dx.doi.org/10.1109/JLT.2003.822152

[11] IR Detectors Catalogue (Vigo System, Ozarow Mazowiecki, Poland, 2006). 